\title{
Relationship among total tear IgE, specific serum lgE, and total serum IgE levels in patients with pollen-induced allergic conjunctivitis
}

\author{
Yasuo Yamana ${ }^{1}$ [D $\cdot$ Satoshi Yamana ${ }^{2}$ Eiichi Uchio ${ }^{3}$
}

Received: 19 March 2021 / Revised: 20 July 2021 / Accepted: 27 July 2021 / Published online: 20 August 2021

(c) The Author(s) 2021

\begin{abstract}
Background Recently, the number of patients with pollinosis, particularly Japanese cedar pollinosis, has markedly increased. We previously reported about local allergic conjunctivitis, which is a phenotype of allergic conjunctivitis (AC). AC cases are often sensitized by various antigens. This study aimed to investigate the relationship among total tear $\operatorname{IgE}(\mathrm{t}-\mathrm{tg} \mathrm{Ig})$, specific serum IgE (s-sIgE), and total serum IgE (t-sIgE) levels in patients with pollen-induced AC.

Methods In 2019, 1372 patients were clinically diagnosed with AC at the Yamana Eye Clinic using t-tIgE, t-sIgE, and s-sIgE tests against 39 allergens. Among the pollen-induced AC patients who underwent allergen testing, 99 tested positives for s-sIgE against pollen. The subjects comprised 33 (33.3\%) male and 66 (66.7\%) female individuals aged 9-86 years.

Results The t-tIgE test was positive in 68 (68.7\%) patients and negative in 31 (31.3\%) patients. In the t-sIgE test, $45(45.5 \%)$ patients had t-sIgE levels above the reference value of $170 \mathrm{IU} / \mathrm{mL}$. The higher the total score of the positive class value of each pollenspecific IgE (pollen-sIgE) antibody, the higher the positive rate of t-tIgE $(p<0.001)$. Of 32 patients in whom food-specific IgE (food-sIgE) was detected, $81.3 \%$ of the pollen-sIgE-positive and food-sIgE-positive cases were also positive for t-sIgE and t-tIgE. However, significant difference was not found between the total score of food-sIgE of the t-tIgE positive group and negative group. Conclusions Pollen-induced AC is caused by pollen sensitization of the conjunctiva. Food-induced AC might be induced by the different pathological mechanism involved in pollen-induced AC.
\end{abstract}

Keywords Pollen-induced allergic conjunctivitis · Food-induced allergic conjunctivitis · Total tear IgE · Specific serum $\operatorname{IgE} \cdot$ Total serum IgE

\section{Key messages}

What is known: The allergen testing of total tear IgE, specific serum IgE and total serum IgE is valuable to evaluate allergic conjunctivitis.

\section{What is new:}

- In many cases of total tear IgE-positive, pollen-specific IgE antibody is higher than other antigens.

- Pollen-induced allergic conjunctivitis can be caused by pollen sensitization in the conjunctiva because pollen-specific IgE antibodies have a higher total tear IgE positive rate than other antigens.

Yasuo Yamana

yamanaganka@yamana.or.jp

Yamana Eye Clinic, Fukuoka, Japan

2 Department of Ophthalmology, Graduate School of Medical

Sciences, Kyushu University, Fukuoka, Japan

3 Department of Ophthalmology, Faculty of Medicine,

Fukuoka University, Fukuoka, Japan 


\section{Introduction}

Recently, the number of patients with pollinosis, particularly Japanese cedar pollinosis, has markedly increased [1, 2]. The antigens that cause allergic conjunctivitis (AC), their sensitization pathways, and pathogenic mechanisms are diverse and are not only sensitized to a single antigen but often to multiple antigens. In addition, allergic diseases have various phenotypes such as local allergic rhinitis and asthma [3-5]. Patients with AC are also sensitized to the conjunctiva by various antigens [3]. We previously reported that $\mathrm{AC}$ has different phenotypes [6].

Aghayan-Ugurluoglu et al. analyzed timothy grass and birch and found allergen-specific $\operatorname{IgA}$ antibodies in the mucosal secretions of patients (e.g., tears) [7]. Ibrahim et al. reported that there was a correlation between specific $\operatorname{IgE}$ in tears and skin in patients with AC [8]. In Japan, Mimura et al. reported that both cedar pollen- and Dermatophagoides pteronyssinus-specific IgE levels in tears were significantly higher in the allergic group than in the control group [9].

We diagnosed and treated $\mathrm{AC}$ according to the Guidelines for the Clinical Management of Allergic Conjunctival Disease of the Japanese Ophthalmology Society (hereafter referred to as "the guidelines") [10]. This study aimed to investigate the relationship among total tear IgE (t-tIgE), specific serum IgE (s-sIgE) against 39 allergens, and total serum IgE (s-tIgE) levels in patients with pollen-induced AC.

Regarding food-induced AC, the Japanese guidelines for food allergy 2020 [11] only describe symptoms such as conjunctival hyperemia, edema, and pruritus for foodinduced AC. Thus, we also investigated the relationship between food- and pollen-induced AC.

\section{Methods}

\section{Study design and participants}

This study followed the ethical principles of the Declaration of Helsinki and was approved by the Ethics Committee of Japan Clinical Society of Diabetes (No. 22/10//2019-6). The study has been registered as the retrospective observational study in the UMIN Clinical Trials Registry (UMIN Trial ID: UMIN000041978). Informed consent was obtained from all individual participants included in this study.

In 2019, 1372 patients obtained a clinical diagnosis of AC based on subjective and clinical symptoms at the Yamana Eye Clinic. The patients who complained of allergic symptoms such as eye itching, discomfort, redness, swelling, eye mucus, nasal discharge, sneezing and stuffy nose, and underwent allergen testing were included. The patients without the results of t-tIgE, s-sIgE, and t-sIgE tests were excluded. There are no age or gender restrictions on the participants.

We have been performing allergen testing for the patients who wish to have them since 2013, and 212 patients underwent allergen testing. Among the 212 patients, 99 (46.7\%) tested positive for s-sIgE against pollen. This study included $33(33.3 \%)$ male and $66(66.7 \%)$ female participants aged 9-86 years (Table 1).

\section{Measurement of allergen testing}

The following tests were performed according to "the guidelines." The t-tIgE levels were measured using the Allerwatch ${ }^{\circledR}$ Tear IgE kit (Wakamoto Pharmaceutical Co., Ltd., Tokyo, Japan) with immunochromatography $[12,13]$. The s-sIgE antibody levels against 39 allergens, including $D$. pteronyssinus, cockroach, cat dander, dog dander, Japanese cedar, orchard grass, ragweed, and mugwort, were measured using the View Allergy $39 \circledR$ kit (Thermo Fisher Diagnostics K.K., Tokyo, Japan) with a fluorescence enzyme immunoassay (FEIA) (Table 2). View Allergy $39{ }^{\circledR}$ kit also utilizes immunochromatography based on a sandwich method but with $\beta$-galactosidase as the labeled substance. Judgment criteria of View Allergy $39 \circledR$ kit are the following: class 1 is false positive and $\geq 0.27$ index; classes 2 to 6 are positive and class 2 is $\geq 0.50$ index; class 3 is $\geq 1.80$ index; class 4 is $\geq 7.05$ index; class 5 is 17.35 index; and class 6 is 29.31 index. The t-sIgE level was measured using FEIA: IgE-RIST (radio-immunosorbent test). The standard value of t-sIgE was $<170 \mathrm{IU} / \mathrm{mL}$ and is the reference range that includes $95 \%$ of healthy people.

\section{Statistical analysis}

Mann-Whitney test and Wilcoxon rank-sum test were used for the comparison of the average between negativity and positivity for t-tIgE. $P$ value less than 0.05 was considered to indicate statistical significance. All statistical analyses were carried out using $\mathrm{R}$ version 3.5.2 software (R Foundation for Statistical Computing, Vienna, Austria).

\section{Results}

\section{Participants}

The participants were $99 \mathrm{AC}$ patients. The data of 99 participants was shown in Table 1. The mean age was 62.6 years; the positivity of t-tIgE was 68 cases, negative in 31 cases, and $\mathrm{t}$-sIgE values of $\geq 170 \mathrm{IU} / \mathrm{mL}$ were 45 cases, $\mathrm{t}$-sIgE values of $<170 \mathrm{IU} / \mathrm{mL}$ in 54 cases. Inhaled specific IgE other than pollen was detected in 66 cases, and food-specific IgE (food-sIgE) was detected in 32 cases. 
Table 1 Characteristic of participants $(n=99)$

\begin{tabular}{|c|c|c|c|c|c|c|c|c|c|}
\hline Age group (years) & Number $(\%)$ & $\begin{array}{l}\text { Total serum IgE } \\
\text { values }(\mathrm{IU} / \mathrm{mL})\end{array}$ & Number & Specific serum $\operatorname{IgE}^{*}$ & Number & Total tear IgE & Number & Sex & Number \\
\hline \multirow[t]{3}{*}{$<10$} & $1(1.0 \%)$ & $<170$ & 1 & Pollen only & 0 & Positive & 1 & Men & 1 \\
\hline & & $\geq 170$ & 0 & Other inhaled & 1 & Negative & 0 & Women & 0 \\
\hline & & & & Food & 0 & & & & \\
\hline \multirow[t]{3}{*}{$10-19$} & $5(5.1 \%)$ & $<170$ & 3 & Pollen only & 1 & Positive & 3 & Men & 3 \\
\hline & & $\geq 170$ & 2 & Other inhaled & 4 & Negative & 2 & Women & 2 \\
\hline & & & & Food & 0 & & & & \\
\hline \multirow[t]{3}{*}{$20-29$} & $2(2.0 \%)$ & $<170$ & 0 & Pollen only & 0 & Positive & 2 & Men & 0 \\
\hline & & $\geq 170$ & 2 & Other inhaled & 2 & Negative & 0 & Women & 2 \\
\hline & & & & Food & 2 & & & & \\
\hline \multirow[t]{3}{*}{$30-39$} & $4(4.0 \%)$ & $<170$ & 1 & Pollen only & 0 & Positive & 3 & Men & 1 \\
\hline & & $\geq 170$ & 3 & Other inhaled & 4 & Negative & 1 & Women & 3 \\
\hline & & & & Food & 2 & & & & \\
\hline \multirow[t]{3}{*}{$40-49$} & $6(6.1 \%)$ & $<170$ & 1 & Pollen only & 1 & Positive & 5 & Men & 1 \\
\hline & & $\geq 170$ & 5 & Other inhaled & 5 & Negative & 1 & Women & 5 \\
\hline & & & & Food & 2 & & & & \\
\hline \multirow[t]{3}{*}{$50-59$} & $10(10.1 \%)$ & $<170$ & 7 & Pollen only & 3 & Positive & 8 & Men & 3 \\
\hline & & $\geq 170$ & 3 & Other inhaled & 7 & Negative & 2 & Women & 7 \\
\hline & & & & Food & 3 & & & & \\
\hline \multirow[t]{3}{*}{$60-69$} & $27(27.3 \%)$ & $<170$ & 18 & Pollen only & 13 & Positive & 14 & Men & 10 \\
\hline & & $\geq 170$ & 9 & Other inhaled & 14 & Negative & 13 & Women & 17 \\
\hline & & & & Food & 5 & & & & \\
\hline \multirow[t]{3}{*}{$70-79$} & $31(31.3 \%)$ & $<170$ & 14 & Pollen only & 8 & Positive & 22 & Men & 12 \\
\hline & & $\geq 170$ & 17 & Other inhaled & 20 & Negative & 9 & Women & 19 \\
\hline & & & & Food & 16 & & & & \\
\hline \multirow[t]{3}{*}{$\geq 80$} & $13(13.1 \%)$ & $<170$ & 9 & Pollen only & 7 & Positive & 10 & Men & 2 \\
\hline & & $\geq 170$ & 4 & Other inhaled & 6 & Negative & 3 & Women & 11 \\
\hline & & & & Food & 2 & & & & \\
\hline \multirow[t]{3}{*}{ Total } & 99 & $<170$ & 54 & Pollen only & 33 & Positive & 68 & Men & 33 \\
\hline & & $\geq 170$ & 45 & Other inhaled & 63 & Negative & 31 & Women & 66 \\
\hline & & & & Food & 32 & & & & \\
\hline
\end{tabular}

${ }^{*}$ There are cases where other inhaled specific IgE and food-specific IgE overlap. The list of allergens which were detected was shown in Table 2

Table 2 Allergens included in the specific IgE View allergy 39 test kit

\begin{tabular}{llll}
\hline Inhaled and other allergens & Food allergens & \\
\hline House dust & Dermatophagoides pteronyssinus, house dust & Egg & Egg, ovomucoid \\
Pets & Cat dander, dog dander & Milk & Milk \\
Insects & Moth, cockroach & Wheat & Wheat \\
Trees & Japanese cedar, Japanese cypress, Alder, black birch & Means/grains/seeds & Peanuts, soybeans, buckwheat, sesame, rice \\
Herbs/greases & Orchard grass, ragweed, mugwort, timothy & Crustaceans & Shrimp/lobster, crab \\
Airborne fungi & Alternaria, Aspergillus & Fruit & Kiwis, apples, bananas \\
Fungi and others & Candida, Malassezia, latex & Fish/meat & Tuna, salmon, mackerel, beef, chicken, pork \\
\hline
\end{tabular}

\section{Result of allergen testing}

The pollen-specific IgE (pollen-sIgE) test elucidated 99 positive cases; however, the proportion of cases in which only pollen-sIgE was detected was 33 cases (33.3\%). In the remaining 66 cases (66.7\%), s-sIgE other than pollen-sIgE was detected.

Regarding t-sIgE and t-tIgE levels according to the class of pollen-sIgE and regarding t-sIgE and t-tIgE levels of 
pollen-sIgE for each class, there were many cases in which pollen-sIgE was high among cases in which t-tIgE was positive and t-sIgE was high (Fig. 1). In cases with high total pollen-sIgE score, the positive rate of t-tIgE was also high $(p<0.001)$ (Fig. 2). Their mean \pm standard deviation (SD) of the total pollen-sIgE score was $3.4 \pm 2.3$ points.

The s-sIgE against allergens other than pollen was detected in 66 cases $(66.7 \%)$. The t-tIgE and t-sIgE test results showed that $68.7 \%$ of pollen-sIgE-positive cases were $\mathrm{t}$-tIgE positive, and $45.5 \%$ of them had $\mathrm{t}$-sIgE values of $\geq 170 \mathrm{IU} / \mathrm{mL}$. In cases where the $\mathrm{t}-\mathrm{sIgE}$ value was higher than the standard value, the proportion of cases in which s-sIgE was detected for allergens other than pollen was 93.3\%.

Tables 3 and 4 show the results of t-sIgE and t-tIgE tests for 32 cases in which food-sIgE was detected. In 32 patients who were food-sIgE positive, $81.3 \%$ of the pollen-sIgEpositive and food-sIgE-positive cases had t-sIgE and t-tIgE above standard levels (Fig. 3).

Regarding the relationship between the total score of food-sIgE and t-tIgE levels, a significant difference was not found between the total score of food-sIgE of the t-tIgE positive group and negative group (Fig. 4).

\section{Discussion}

In "the guidelines" [10], AC is defined as non-proliferative conjunctivitis associated with a type I allergy that is mediated by IgE antibodies. In this study, we examined the relationship among t-tIgE, t-sIgE, and s-sIgE in patients with pollen-induced AC.
A commercial kit, Allerwatch ${ }^{\circledR}$ Tear $\operatorname{IgE}$ [12-15], that measures total IgE antibody levels in lachrymal fluids has recently become available, and it confirms the local production of $\operatorname{IgE}$ antibodies in the eye.

Aghayan-Ugurluoglu et al. [7] searched for birch and timothy grass pollen allergen-specific IgA antibodies in tear fluids and reported that "serum and tears of many of the pollen-allergic individuals with conjunctivitis exhibited specificity for the very same pollen allergens."

Ibrahim et al. [8] noted that serum and tears of many pollen-allergic individuals with conjunctivitis exhibited specificity for the same pollen allergens. Hoffmann-Sommergruber et al. [16] investigated whether the presence of $\mathrm{IgE}$ in tears of grass pollen-allergic patients correlated with the disease and clinical symptoms. They concluded that allergen-specific IgE antibodies in tears seem to be produced locally rather than as serum exudate and that IgE in tears seems to be responsible for AC. In Japan, Mimura reported that both cedar pollen- and D. pteronyssinus-specific IgE levels in tears were significantly higher in the allergic group than in the control group [9]. From these reports, it may be speculated that pollen-induced AC is sensitized by the conjunctiva. In general, allergic patients may be sensitized to multiple antigens to varying degrees. However, until now, no reports have investigated the relationship between various antigens and pollen-induced AC.

Based on the current study results, the t-sIgE positivity rate was as low as $33.3 \%$ in the case of pollen-sIgE alone. Moreover, the positivity for both pollen-sIgE and non-pollen-sIgE was $66.7 \%$, which was relatively low. For the t-tIgE positivity rate, even in cases with positivity to only pollenSIgE, the t-tIgE positivity rate was $63.6 \%$, but only $9.1 \%$ of cases had high t-sIgE levels, which was low. Furthermore,
Fig. 1 Total serum IgE and total tear IgE (t-tIgE) levels according to the class of pollenspecific IgE (pollen-sIgE). The total serum IgE reference value was less than $170 \mathrm{IU} / \mathrm{mL}$. Class 1 is false positive and $\geq 0.27$ index; class 2 is $\geq 0.50$ index; class 3 is $\geq 1.80$ index; class 4 is $\geq 7.05$ index; class 5 is 17.35 index; class 6 is 29.31 index. Classes 2 to 6 are positive

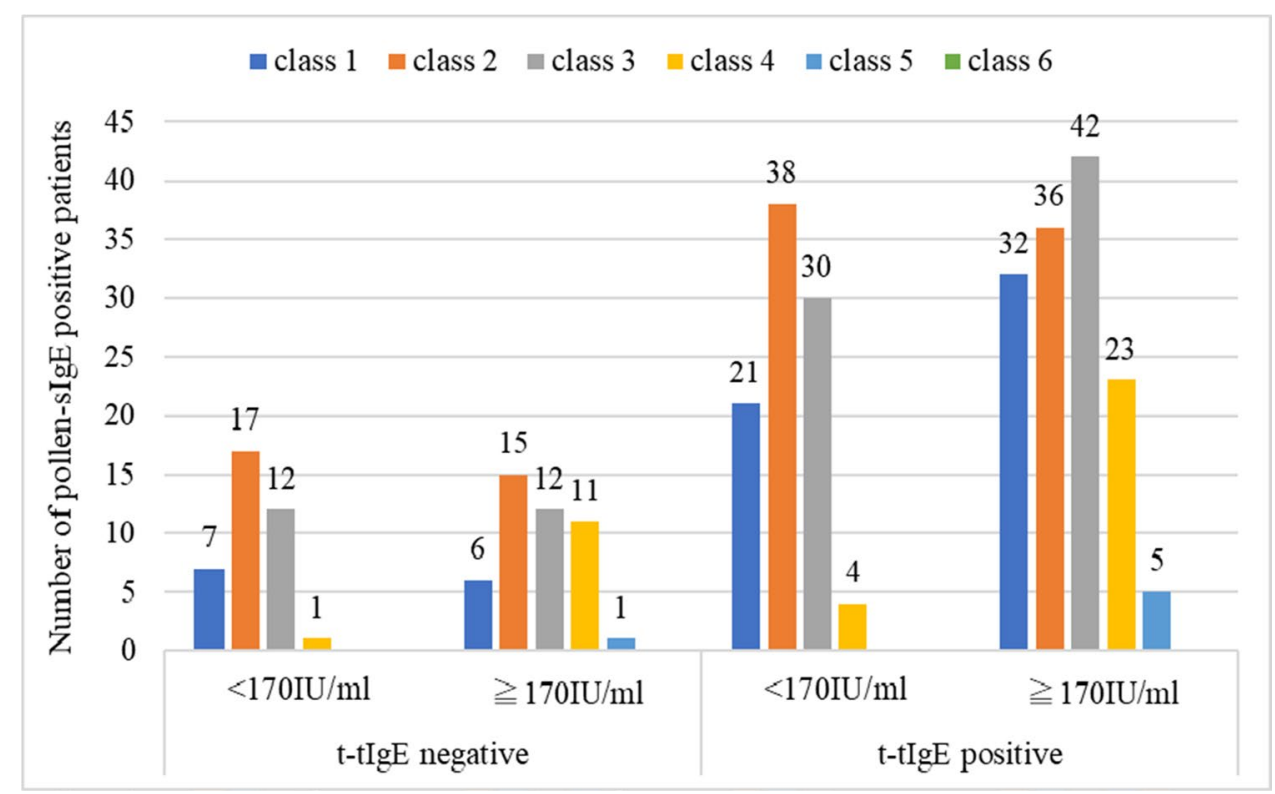


Fig. 2 Relationship between the total score of pollen-specific IgE (pollen-sIgE) and total tear IgE levels

Table 3 Results of the total serum IgE test for 32 cases in which food-specific IgE was detected

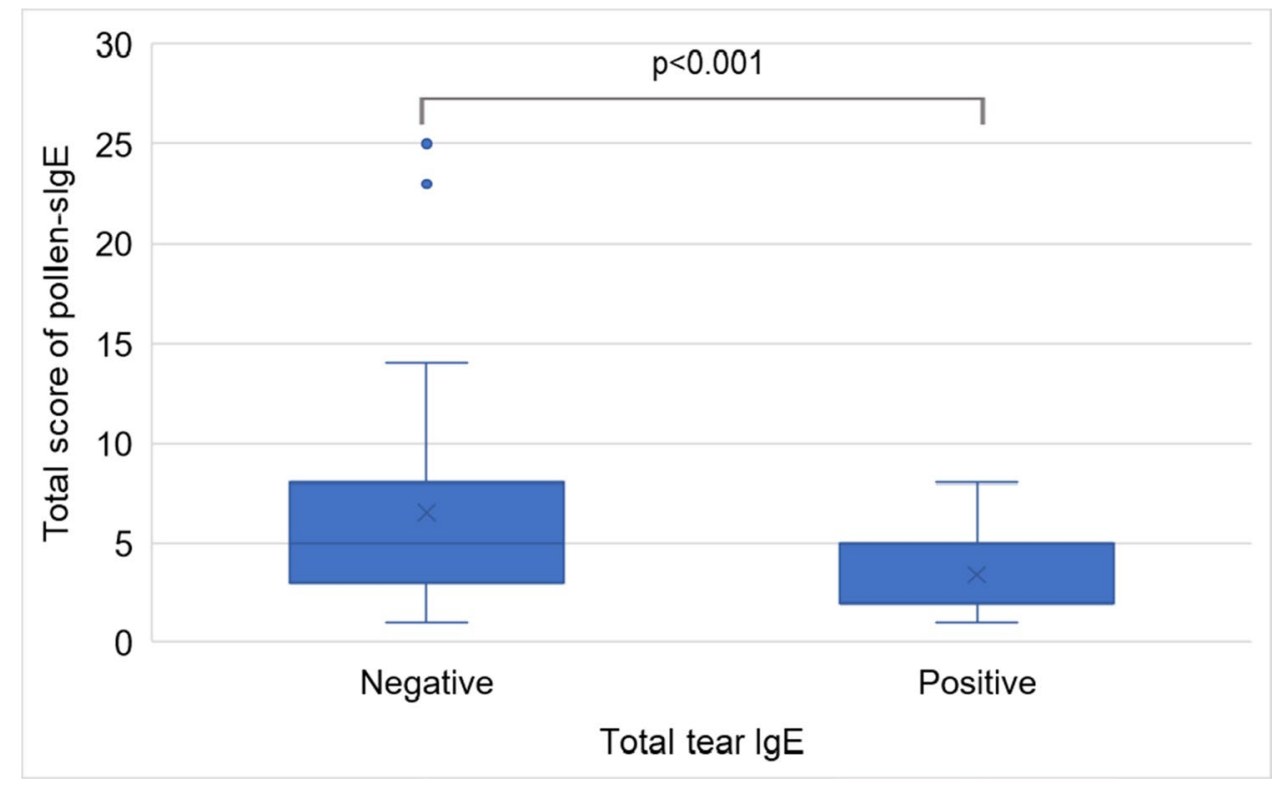

\begin{tabular}{lll}
\hline & \multicolumn{2}{l}{ Total serum IgE } \\
\cline { 2 - 3 } \cline { 3 - 3 } & $<170 \mathrm{IU} / \mathrm{mL}$ & $\mathbf{2}$ \\
\hline Animal food-specific IgE only & 3 & 12 \\
Plant-derived food-specific IgE only & 2 & 8 \\
Both animal and plant-derived food-specific IgE & 1 & 26 \\
Food-specific IgE & 6 & \\
\hline
\end{tabular}

Table 4 Results of the total tear IgE test for 32 cases in which foodspecific IgE was detected

\begin{tabular}{lll}
\hline & $\begin{array}{l}\text { t-tIgE } \\
\text { nega- } \\
\text { tive }\end{array}$ & t-tIgE positive \\
\hline Animal food-specific IgE only & 2 & 7 \\
Plant-derived food-specific IgE only & 1 & 13 \\
Both animal and plant-derived food-specific & 3 & 6 \\
IgE & 6 & 26 \\
Food-specific IgE & 6 & \\
\hline
\end{tabular}

$t$-tIgE, total tear IgE

for $\mathrm{t}$-sIgE and t-tIgE levels of pollen-sIgE for each class, there were many significant differences among cases in which $\mathrm{t}$-tIgE was positive and $\mathrm{t}$-sIgE was high, and the class of pollen-sIgE was also high.

These results suggest that pollen-induced AC is first sensitized by the conjunctiva. However, the power of sensitization was considered weak with only a single pollen antigen. In addition, it could be inferred that non-pollen-sIgE was sensitized in cases where the t-sIgE level was higher than the standard value.
Next, the relationship between pollen-induced AC and food-induced $\mathrm{AC}$ was investigated. Regarding the relationship between food and AC, Mimura et al. [17] stated that wheat allergy is involved in AC. Pollen and food allergies together are known as pollenfood allergy syndrome $[18,19]$. This description is not found in the Japanese Food Allergy Guideline 2020 [11], which only explains clinical symptoms such as conjunctival hyperemia and edema.

In this study, $81.3 \%$ of pollen-sIgE-positive and foodsIgE-positive cases were associated with $\mathrm{t}$-sIgE and $\mathrm{t}$-tIgE above standard levels. Furthermore, $81.3 \%$ of pollen-sIgEpositive and food-sIgE-positive cases showed higher values than the standard t-sIgE and t-tIgE values (Fig. 3). Mimura et al. reported regarding the correlation between the t-tIgE score and s-sIgE levels against house dust mites and found that house dust mite allergens may be the primary cause of AC during autumn in Japan [20]. Despite these reports, the lack of significant difference of the total score of food-sIgE between the t-tIgE positive group and negative group suggests that food-induced AC might be induced by the different pathological mechanism involved in pollen-induced AC. 
Fig. 3 Results of total tear $\operatorname{IgE}$ and total serum IgE tests for 32 cases in which food-specific IgE was detected. The total serum $\operatorname{IgE}$ reference value was less than $170 \mathrm{IU} / \mathrm{mL}$. Among the cases showing positivity for pollen-specific IgE and foodspecific $\operatorname{IgE}$, the total serum $\operatorname{IgE}$ and total tear $\operatorname{IgE}$ levels were higher than the standard levels in $81.3 \%$ of the cases. Animalderived food IgE includes egg, ovomucoid, milk, shrimp/lobster, crab, tuna, salmon, mackerel, beef, chicken, and pork. Plant-derived food IgE includes wheat, peanuts, soybeans, buckwheat, sesame, rice, kiwis, apple, and bananas

Fig. 4 Relationship between the total score of food-specific $\operatorname{IgE}$ (food-sIgE) and total tear IgE levels
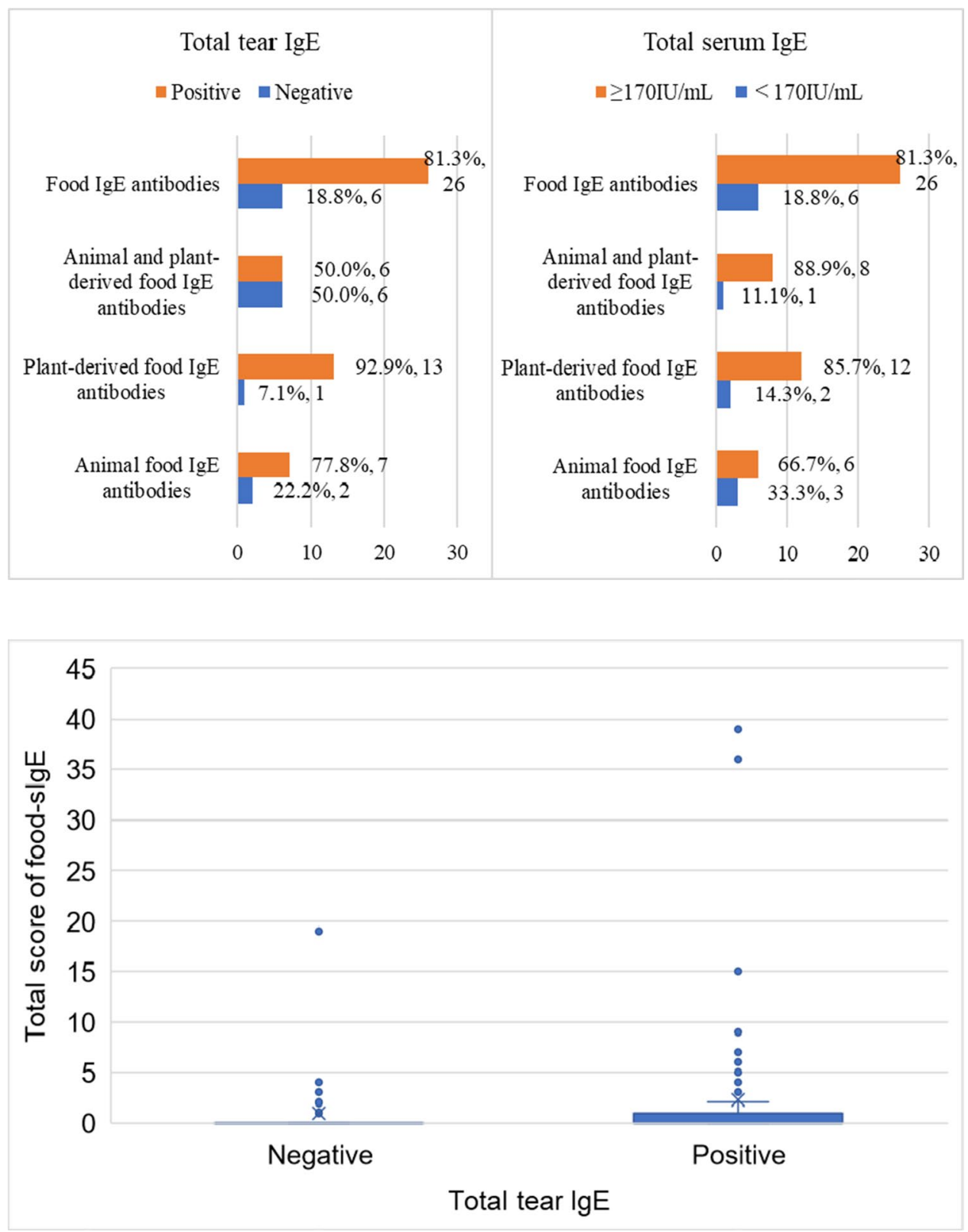

In conclusion, based on the high proportion of cases with high pollen-sIgE-positive scores among t-tIgE-positive cases, pollen-induced AC is very likely caused by pollen sensitization of the conjunctiva.

Abbreviations AC: Allergic conjunctivitis; IgE: Immunoglobulin E; s-sIgE: Specific serum IgE; s-tIgE: Total serum IgE; t-tIgE: Total tear IgE; View39: View allergy 39 kit; RIST: Radio-immunosorbent test; FELA: Fluorescence enzyme immunoassay; pollen-sIgE: Pollen-specific IgE; food-sIgE: Food-specific IgE; D. pteronyssinus: Dermatophagoides pteronyssinus

Acknowledgements The authors would like to thank Kenji Okamoto of Softcare Co., Ltd. for data analysis.
Author contribution Y.Y. designed the study and performed experiments. Y.Y., Y.S., and E.U. wrote the manuscript. E.U. performed the statistical analysis. All authors read and approved the final manuscript.

\section{Declarations}

Ethics approval All procedures performed in studies involving human participants were in accordance with the ethical standards of the ethical committee of Japan clinical Society of Diabetes which is a closed association of 145 practicing diabetes specialists (NO.22/10/2019-6) and with the 1964 Helsinki Declaration and its later amendments or comparable ethical standards.

Informed consent Informed consent was obtained from all individual participants included in the study. 
Conflicts of interest The authors declare no competing interests.

Open Access This article is licensed under a Creative Commons Attribution 4.0 International License, which permits use, sharing, adaptation, distribution and reproduction in any medium or format, as long as you give appropriate credit to the original author(s) and the source, provide a link to the Creative Commons licence, and indicate if changes were made. The images or other third party material in this article are included in the article's Creative Commons licence, unless indicated otherwise in a credit line to the material. If material is not included in the article's Creative Commons licence and your intended use is not permitted by statutory regulation or exceeds the permitted use, you will need to obtain permission directly from the copyright holder. To view a copy of this licence, visit http://creativecommons.org/licenses/by/4.0/.

\section{References}

1. Kaneko Y, Motohashi Y, Nakamura H, Endo T, Eboshida A (2005) Increasing prevalence of Japanese cedar pollinosis: a metaregression analysis. Int Arch Allergy Immunol 136(4):365-371. https://doi.org/10.1159/000084256

2. Okubo K, Kurono Y, Ichimura K, Enomoto T, Okamoto Y, Kawauchi H, Suzaki H, Fujieda S, Masuyama K, Japanese society of allergology, (2020) Japanese guidelines for allergic rhinitis. Allergol Int 69(3):331-345. https://doi.org/10.1016/j.alit.2020.04. 001

3. Novak N, Bieber T (2003) Allergic and nonallergic forms of atopic diseases. J Allergy Clin Immunol 112(2):252-262. https:// doi.org/10.1067/mai.2003.1595

4. Rondón C, Fernández J, López S, Campo P, Doña I, Torres MJ, Mayorga C, Blanca M (2009) Nasal inflammatory mediators and specific IgE production after nasal challenge with grass pollen in local allergic rhinitis. J Allergy Clin Immunol 124(5):1005-11. e1. https://doi.org/10.1016/j.jaci.2009.07.018

5. Eguiluz-Gacia I, Ariza A, Testera-Montes A, Rondón C, Campo P (2020) Allergen immunotherapy for local respiratory allergy. Curr Allergy Asthma Rep 20:23. https://doi.org/10.1007/ s11882-020-00920-w

6. Yamana Y, Fukuda K, Ko R, Uchio E (2019) Local allergic conjunctivitis: a phenotype of allergic conjunctivitis. Int Ophthalmol 39:2539-2544. https://doi.org/10.1007/s10792-019-01101-z

7. Aghayan-Ugurluoglu R, Ball T, Vrtala S, Schweiger C, Kraft D, Valenta R (2000) Dissociation of allergen-specific $\operatorname{IgE}$ and $\operatorname{Ig} \mathrm{A}$ responses in sera and tears of pollen-allergic patients: a study performed with purified recombinant pollen allergens. J Allergy Clin Immunol 105(4):803-813. https://doi.org/10.1067/mai.2000. 104782

8. Ibrahim BM, Abdel-Latif RS (2017) Correlation of tear film-specific immunoglobulin $\mathrm{E}$ assay with the skin prick test in allergic conjunctivitis. Delta J Ophthalmol 18(3):123-132. https://doi.org/ 10.4103/DJO.DJO_30_17

9. Mimura T, Yamagami S, Kamei Y, Goto M, Matsubara M (2013) Specific IgE in tear fluid and features of allergic conjunctivitis. Curr Eye Res 38(9):917-925. https://doi.org/10.3109/02713683. 2013.794248

10. Miyazaki D, Takamura E, Uchio E, Ebihara N, Ohno S, Ohashi Y, Okamoto S, Satake Y, Shoji J, Namba K, Fukagawa K, Fukushima
A, Fujishima H; Japanese Society of Ovular Allergology; Japanese Society of Allergology (2020) Japanese guidelines for allergic conjunctival diseases 2020. Allergol Int 69(3): 346-55.https:// doi.org/10.1016/j.alit.2020.03.005

11. Ebisawa M, Ito K, Fujisawa T (2020) Japanese guidelines for food allergy 2020. Allergol Int 69(3):370-386. https://doi.org/ 10.1016/j.alit.2020.03.004

12. Inada N, Shoji J, Kato H, Kiely S, Mulyanto SM (2009) Clinical evaluation of total IgE in tears of patients with allergic conjunctivitis disease using a novel application of the immunochromatography method. Allergol Int 58(4):585-589. https://doi.org/10. 2332/allergolint.09-OA-0101

13. Mimura FT, Usui T, Mori M, Funatsu H, Noma H, Aixinjueluo W, Yamamoto H, Amano S (2010) Rapid immunochromatography of total tear immunoglobulin $\mathrm{E}$ in allergic conjunctivitis with Allerwatch. J Investig Allergol Clin Immunol 20(7):627-628

14. Mimura T, Usui T, Yamagami S, Miyai T, Amano S (2013) Relationship between total tear IgE and specific serum IgE in autumnal allergic conjunctivitis. Cornea 32(1):14-19. https://doi.org/ 10.1097/ICO.0B013e318253214b

15. Mimura T, Usui T, Yamagami S, Miyai T, Amano S (2012) Relationship between total tear $\operatorname{IgE}$ and severity of acute seasonal allergic conjunctivitis. Curr Eye Res 37(10):864-870. https://doi. org/10.3109/02713683.2012.689069

16. Hoffmann-Sommergruber K, Ferreira ED, Ebner C, Barisani T, Korninger L, Kraft D, Scheiner O, Baumgartner I (1996) Detection of allergen-specific IgE in tears of grass pollen-allergic patients with allergic rhinoconjunctivitis. Clin Exp Allergy 26(1):79-87. https://doi.org/10.1111/j.1365-2222.1996.tb00059.x

17. Mimura T, Yamagami S, Noma H, Kamei Y, Goto M, Kondo A, Matsubara M (2015) Specific IgE for wheat in tear fluid of patients with allergic conjunctivitis. Cutan Ocul Toxicol 35(1):25-34. https://doi.org/10.3109/15569527.2014.890938

18. Kim MA, Kim DK, Yang HJ, Yoo Y, Ahn Y, Park HS, Lee HJ, Jeong YY, Kim BS, Bae WY, Jang AS, Park Y, Koh YI, Lee J, Lim DH, Kim JH, Lee SM, Kim YM, Jun YJ, Kim HY, Kim Y, Choi JH; Work Group for Rhinitis, the Korean Academy of Asthma, Allergy and Clinical Immunology (2018) Pollen-food allergy syndrome in Korean pollinosis patients: a nationwide survey. Allergy Asthma Immunol Res 10(6):648-61.https://doi.org/ 10.4168/aair.2018.10.6.648

19. Mastrorilli C, Tripodi S, Caffarelli C, Perna S, Di Rienzo-Businco A, Sfika I, Asero R, Dondi A, Bianchi A, PovesiDascola C, Ricci G, Cipriani F, Maiello N, Miraglia Del Giudice M, Frediani T, Frediani S, Macrì F, Pistoletti C, Dello Iacono I, Patria MF, Varin E, Peroni D, Comberiati P, Chini L, Moschese V, Lucarelli S, Bernardini R, Pingitore G, Pelosi U, Olcese R, Moretti M, Cirisano A, Faggian D, Travaglini A, Plebani M, Verga MC, Calvani M, Giordani P, Matricardi PM, Allergy IP, Network(I-PAN), (2016) Endotypes of pollen-food syndrome in children with seasonal allergic rhinoconjunctivitis: a molecular classification. Allergy 71(8):1181-1191. https://doi.org/10.1111/all.12888

20. Mimura T, Usui T, Mori M, Funatsu H, Noma H, Amano S (2011) Specific tear IgE in patients with moderate-to-severe autumnal allergic conjunctivitis. Int Arch Allergy Immunol 156(4):381386. https://doi.org/10.1159/000323908

Publisher's note Springer Nature remains neutral with regard to jurisdictional claims in published maps and institutional affiliations. 